Les mathématiques dans les écoles militaires 



\title{
Introduction. Les mathématiques dans les écoles militaires
}

\author{
Mónica Blanco \\ Departament de Matemàtiques, \\ Universitat Politècnica de Catalunya, \\ BarcelonaTech (Espagne)
}

Olivier Bruneau Archives Henri-Poincaré - Philosophie et Recherches sur les Sciences et les Technologies (AHP-PReST), Université de Lorraine, CNRS, Université de Strasbourg, Nancy (France)

Aborder l'histoire des mathématiques et son enseignement à travers les institutions scientifiques est une démarche dorénavant courante et souvent pertinente. De nombreux travaux l'ont montré en particulier dans le cadre des grandes institutions scientifiques militaires comme l'École polytechnique [Belhoste, Dahan Dalmedico et al. 1994], [Bret 2002]. L'histoire de l'enseignement et de la diffusion des sciences, en particulier des mathématiques, a été renouvelée depuis une vingtaine d'années tant en France que dans d'autres pays européens ou américains ${ }^{1}$. Par ailleurs, le rôle des écoles militaires dans la production des mathématiques est connu ${ }^{2}$. L'École polytechnique et son école d'application du génie et d'artillerie de Metz ont une place centrale dans le développement des mathématiques au début du XIX ${ }^{\mathrm{e}}$ siècle [Gillispie 1994].

Il est aussi à noter que les diverses académies militaires ont été créées relativement au même moment : les écoles régimentaires d'artillerie et l'Académie royale militaire de mathématiques de Barcelone en 1720, la Royal Military Academy à Woolwich en 1741, l'École du génie militaire en 1748

Philosophia Scientice, 24(1), 2020, 5-11.

1. Voir par exemple [Olesko 2006], [Karp \& Schubring 2014].

2. La littérature est abondante, on pourra se référer à [Bruneau 2015], [Guicciardini 1989], [Massa-Esteve, Roca-Rosell et al. 2011], [Navarro Loidi 2013], [Preveraud 2013, 2014], parmi d'autres. 
à Mézières, l'École militaire à Paris et l'Académie mathématique des gardes royaux à Madrid en 1750, puis plus tardivement l'École polytechnique en 1794 et le Royal Military College en Angleterre en 1801, l'Académie militaire de West Point en 1802.

Ces différentes écoles subissent des évolutions dans leurs statuts, dans leur mode de recrutement ou dans la fusion de la formation des corps génie et artillerie (civil et militaire). Il apparaît aussi des changements dans les curricula (comme, par exemple, l'incorporation de l'enseignement de la géométrie descriptive). Ces modifications ont des origines diverses. Elles peuvent être à la suite de défaites cuisantes, d'une volonté de remettre au goût du jour divers enseignements, d'une volonté de remettre en phase les exigences du terrain (ou du champ de bataille) [Alfonsi \& Guilbaud 2015] ou la formation des officiers [Galland Seguela 2008].

Dans l'historiographie, ces évolutions ont quasi-exclusivement été étudiées isolément dans une perspective locale ou nationale. Il n'existe pas d'étude qui embrasse ces questions dans une vision globale, en comparant finement ces évolutions à travers le prisme transnational, en évaluant les différents freins ou obstacles locaux dans le cadre pédagogique, institutionnel, politique ou diplomatique.

Dans ce dossier thématique, nous interrogeons les dynamiques locales ou nationales afin de voir si cela provoque des modifications substantielles sur la place des mathématiques et de leur enseignement dans les écoles militaires. De plus, nous examinons les transferts du savoir mathématique entre les différentes écoles militaires au sein des pays mais aussi par-delà les frontières. Par ailleurs, nous étudions si, parallèlement ou de façon indépendante, il existe des changements dans les programmes d'enseignement des mathématiques, c'est-à-dire si lorsque dans une école il y a une évolution de curriculum, d'autres écoles nationales ou étrangères opèrent ce même changement ou si elles préfèrent conserver un enseignement plus traditionnel.

Ce dossier thématique propose des études de cas sur l'enseignement des mathématiques dans les écoles militaires, à travers des contextes nationaux divers, comme en Colombie, en Espagne, au Royaume-Uni, aux États-Unis, aux Pays-Bas et en France. Pour aborder ces études de cas, nous mettons l'accent sur le lien entre le local et le global en nous appuyant sur une analyse comparative et d'une étude des réseaux de communication dans une perspective internationale. Pour cela, il faut donc s'appuyer sur une étude du local-national, sans perdre de vue le contexte international avec une perspective transnationale, tout en considérant les concepts clés comme la comparaison, la communication et l'appropriation [Simon \& Herran 2008, 6-7].

D'abord, l'analyse comparative, nationale et même transnationale, porte sur des objets potentiellement comparables. Dans ce dossier thématique on insiste sur les dimensions suivantes : 
1. les curricula, les manuels utilisés et les concours d'admission,

2. les liens existant entre les enseignements mixtes génie-artillerie et civilmilitaire,

3. le rôle des mathématiques dans l'enseignement, en particulier celui de la géométrie descriptive et de l'analyse.

Par ailleurs, l'étude de la transmission s'appuie sur les transferts des savoirs, des manuels et de leurs traductions ainsi que sur les "voyageurs » - les praticiens qui ont franchi des frontières nationales. L'attention se déplace donc vers le public et l'usage, au lieu de l'origine et de la production [Secord 2004].

Finalement, l'analyse de l'appropriation des objets mentionnés ci-dessus, à travers des réseaux de communication, sert à structurer ce dossier thématique autour de deux axes. Le premier axe est le discours centre-périphérie. La production du savoir scientifique est plus complexe que la simple réception et reproduction du savoir et il faut prendre en compte les différentes nuances mises en jeu. Le cadre conceptuel centre-périphérie est souvent représenté par un modèle statique avec une transmission du centre vers la périphérie - dans le cas de la France avec l'École polytechnique au centre et ses différentes écoles d'application à la périphérie. Il faut donc re-questionner les stratégies de construction et de communication des savoirs scientifiques pour redéfinir ce qu'est le concept de «périphérie » [Guillem-Llobat 2008]; [Nieto-Galan 2008]. Un courant historiographique récent met l'accent plutôt sur l'appropriation qui est du registre de l'action, s'éloignant ainsi de l'idée de transmission vue comme une réception passive. Une analyse comparative transnationale peut aider à revoir le cadre centre-périphérie monolithique susmentionné [Cohen \& O'Connor 2004], [Simon \& Herran 2008].

Les articles de M. Ángeles Velamazán \& Elena Ausejo, de Bertrand Eychenne, de Thomas Preveraud et de Yannick Vincent abordent cette question.

M. A. Velamazán et E. Ausejo montrent comment l'École polytechnique et l'École d'application de l'artillerie et du génie de Metz ont influencé l'Académie d'ingénieurs militaires espagnole en tant que modèles en s'appuyant sur des plans d'études et des manuels mathématiques tout au long du $\mathrm{XIX}^{\mathrm{e}}$ siècle. Cette volonté nette de suivre des modèles français s'explique par la volonté d'augmenter le niveau mathématique des ingénieurs militaires. Si cet article parle du cadre centre-périphérie, celui-ci n'est pas si monolithique tel que considéré traditionnellement.

B. Eychenne présente un cas où l'influence du centre sur la périphérie n'est pas si évidente qu'il n'y paraît. Après l'accession de la Colombie à l'indépendance, le défi de la mise en place d'un enseignement scientifique et technique trouve finalement sa résolution en 1848 avec la création d'une école militaire, le Colegio Militar. L'étude de cet établissement implanté sur le continent sud-américain dans un contexte postcolonial, permet à B. Eychenne 
de porter un regard décentré et précis sur les spécificités locales d'une institution militaire d'enseignement des mathématiques, tout en montrant les choix opérés et l'influence des contextes politiques et sociaux. L'enjeu de l'étude institutionnelle est alors de mettre en évidence la diversité de ces circulations des savoirs, les processus d'adaptation qui les accompagnent, mais surtout la manière dont ceux-ci se répercutent sur les enseignements et leur évolution. B. Eychenne s'écarte des schémas classiques d'organisation des enseignements et prend en compte les influences des contextes politiques et sociaux. Il met l'accent sur les complexités des circulations (dans tous les sens), sur l'appropriation plutôt que sur un système transmission-réception.

Th. Preveraud offre un autre cas centre-périphérie « outre-mer ». Au début du XIX $\mathrm{X}^{\mathrm{e}}$ siècle, la formation des officiers de l'armée des États-Unis s'effectue à l'Académie militaire de West Point. Défaillante en de nombreux points, y compris sur le terrain de l'enseignement mathématique, elle est transformée par Sylvanus Thayer en 1817, alors qu'il revient d'un séjour en Europe lors duquel les établissements militaires français ont fait l'objet de scrupuleuses observations. La supériorité des méthodes françaises - en ce qui concerne les curricula, le rôle de la géométrie descriptive et l'analyse dans l'appréhension de l'art de la guerre et l'usage des manuels - semble chose donnée pour acquise du côté américain, si bien que, dans le sillage de West Point, d'autres académies militaires « à la française » ouvrent avant-guerre. Soutenant l'idée d'une «infusion française » plus ou moins passive, l'historiographie alimente bien souvent un récit qui mythifie et cristallise le rôle de la France dans la formation mathématique des militaires américains de la première moitié $\mathrm{du} \mathrm{XIX}^{\mathrm{e}}$ siècle, un récit qui minore, de fait, par son caractère univoque, l'importance des espaces intermédiaires - scientifiques, pédagogiques, institutionnels et éditoriaux - où entrent en contact les territoires émetteurs et récepteurs. Il s'agit d'un processus de décomposition et de réassemblage des savoirs et méthodes français, dont le «transfert » depuis l'autre côté de l'Atlantique et au sein du territoire américain emprunte des circuits moins prétendument polarisés et hiérarchisés, et très fortement soumis à une forme de rationalisation locale.

Y. Vincent explore le rapport entre l'enseignement et la recherche au sein de l'École polytechnique. L'auteur entend re-questionner l'idée que les acteurs de cette École ne travaillaient pas sur des mathématiques originales. À l'aide de trois exemples de théorèmes attribués à trois enseignants de cette école, il montre comment dans cette structure bien établie, l'enseignement s'y pratique et des savoirs nouveaux s'y propagent.

Puisque les contextes nationaux peuvent être vraiment hétérogènes, le second axe explore les discontinuités ou les ruptures dans des contextes nationaux apparemment unifiés [Gavroglu, Patiniotis et al. 2008], [Simon 2013]. C'est le fil conducteur des articles de Danny Beckers, Mónica Blanco et Carles Puig-Pla, Olivier Bruneau et Juan Navarro Loidi. 
D. Beckers analyse les changements subis par les mathématiques dans la formation des officiers de l'armée néerlandaise au cours du XIX ${ }^{\mathrm{e}}$ siècle. Ces changements sont visibles dans les programmes de l'Académie militaire, fondée en 1828. Les mathématiques dans l'éducation militaire ont une fonction purement pédagogique et la recherche n'est pas encouragée. Il montre que l'Académie était un des lieux les plus importante de diffusion du savoir mathématique aux Pays-Bas dans la première moitié du $\mathrm{XIX}^{\mathrm{e}}$ siècle, mais qu'elle a perdu ce rôle pendant les années 1860-1870. Il s'appuie pour cela sur une étude des programmes et sur le rôle des instructeurs de mathématiques de l'Académie au sein de la Société mathématique et dans l'Académie royale des sciences. L'évolution des mathématiques qui est visible dans ces deux institutions n'est pas la conséquence d'une adaptation du programme de l'Académie. Ainsi dès 1870, on peut considérer que l'Académie a presque complètement disparu du paysage mathématique néerlandais.

M. Blanco et C. Puig-Pla se centrent sur deux études de cas. Vers la fin de l'année 1750, une Académie de mathématiques est créée au sein de l'Académie militaire de la garde du corps à Madrid, dirigée par Pedro Padilla jusqu'à sa fermeture en 1760. En 1753, Padilla commence à publier son Cours militaire de mathématiques (1753-1756) pour un usage dans cette académie. Le besoin de textes mathématiques en espagnol dans le domaine militaire conduit à la création en 1757 de la Société royale militaire de mathématiques à Madrid sous la direction de Pedro de Lucuce, également directeur de l'Académie militaire de mathématiques de Barcelone depuis 1738. Les membres de la Société sont chargés de l'élaboration d'un cours exhaustif dans lequel les mathématiques sont considérées comme essentielles à la formation militaire des artilleurs et des ingénieurs. Le but de cette contribution est d'explorer et de comparer la manière dont l'étude des mathématiques a été abordée dans ces deux cas, dans un contexte d'unité accordé par l'Académie militaire de mathématiques de Barcelone.

O. Bruneau aborde un autre cas de discontinuité au sein d'un contexte de continuité. En 1741, la Royal Military Academy de Woolwich est créée par le Board of Ordnance afin d'instruire les futurs officiers artilleurs et ingénieurs militaires. Cette instruction s'appuie dès le départ sur les mathématiques. Il présente et étudie les différents programmes sur la longue période (entre 1741 et les années 1860). Les évolutions, les changements mais aussi les constances sont évalués et il donne les raisons de ceux-ci. L'âge de recrutement, le poids $\mathrm{du}$ Board of Ordnance ou encore les diverses guerres ont aussi une influence importante sur la place des mathématiques dans les programmes. Par ailleurs, le peu de turn-over des professeurs entraîne aussi une inertie de l'enseignement des mathématiques.

J. Navarro Loidi étudie l'enseignement des mathématiques au Collège d'Artillerie espagnol de 1764 à 1842 et son influence étrangère. On observe qu'après quelques hésitations, un programme standard est adopté commençant par l'arithmétique et finissant par le calcul différentiel et intégral et la mécanique. L'unique changement important fut en 1819 quand la mécanique 
devint une matière indépendante. Pendant les premières décennies, avec Giannini comme premier professeur, l'emprise italienne est importante, mais il existe aussi une influence française et anglaise. Avec le temps c'est l'inspiration française qui supplante toutes les autres.

En résumé, l'objectif de ce dossier est d'étudier l'existence de porosités transnationales dans la production et l'enseignement des mathématiques dans les écoles militaires qu'elles soient européennes ou américaines (du Nord ou du Sud). Par ailleurs, ce dossier sera aussi l'occasion de pointer au sein de ces académies militaires les moments de continuité ou de rupture.

\section{Bibliographie}

Alfonsi, Liliane \& Guilbaud, Alexandre [2015], La guerre de Sept Ans (17561763) et ses conséquences pour les écoles militaires françaises, dans Sciences mathématiques 1750-1850 : continuités et ruptures, édité par Ch. Gilain \& A. Guilbaud, Paris : CNRS Éditions, 127-154.

Belhoste, Bruno, Dahan Dalmedico, Amy et al. (éds.) [1994], La Formation polytechnicienne 1794-1994, Paris : Dunod.

Bret, Patrice [2002], L'Etat, l'armée, la science : L'invention de la recherche publique en France (1763-1830), Rennes : Presses Universitaires de Rennes.

Bruneau, Olivier [2015], La géométrie en Grande-Bretagne 1750-1830, dans Sciences mathématiques 1750-1850 : continuités et ruptures, édité par Ch. Gilain \& A. Guilbaud, Paris : CNRS Éditions, 403-440.

Cohen, Deborah \& O'Connor, Maura [2004], Comparison and History : Europe in cross-national perspective, New York; Londres : Routledge.

Galland Seguela, Martine [2008], Les Ingénieurs militaires espagnols de 1710 à 1803 : étude prosopographique et sociale d'un corps d'élite, Madrid: Casa Velázquez.

Gavroglu, Kostas, Patiniotis, Manolis et al. [2008], Science and technology in the European periphery : Some historiographical reflections, History of Science, 46(2), 153-175, doi : 10.1177/007327530804600202.

Gillispie, Charles [1994], Un enseignement hégémonique : les mathématiques, dans La Formation polytechnicienne 1794-1994, édité par B. Belhoste, A. Dahan Dalmedico \& A. Picon, Paris : Dunod, 31-44.

Guicciardini, Niccolò [1989], The Development of Newtonian Calculus in Britain 1700-1800, Cambridge : Cambridge University Press. 
Guillem-Llobat, Ximo [2008], Science in the periphery, dans Beyond Borders : Fresh perspectives in history of science, édité par J. Simon, N. Herran, T. Lanuza-Navarro, P. Ruiz-Castell \& X. Guillem-Llobat, Cambridge : Cambridge Scholars Press, 291-299.

Karp, Alexander \& Schubring, Gert (éds.) [2014], Handbook on the History of Mathematics Education, New York : Springer.

Massa-Esteve, M. Rosa, Roca-Rosell, Antoni et al. [2011], "Mixed" mathematics in engineering education in Spain : Pedro Lucuce's course at the Barcelona Royal Military Academy of Mathematics in the eighteenth century, Engineering Studies, 3(3), 233-253, doi : 10.1080/19378629.2011. 618188.

Navarro Loidi, Juan [2013], Don Pedro Giannini o las matemáticas de los artilleros del siglo XVIII, Segovia : Asociación Cultural Ciencia y Artillería.

Nieto-Galan, Agusti [2008], The sistory of science in Spain. A critical overview, Nuncius, XXIII(2), 211-236.

Olesko, Kathryn M. [2006], Science pedagogy as a category of historical analysis : Past, present, and future, Science $\&$ Education, 15(7), 863-880, doi : 10.1007/s11191-005-2014-8.

Preveraud, Thomas [2013], Transmissions des enseignements mathématiques français à l'Académie militaire américaine de West Point (1815-1836), Amnis, Revue de civilisation contemporaine Europe/Amériques, 12, en ligne, doi : 10.4000/amnis.1943.

[2014], Circulations mathématiques franco-américaines (1818-1878), Thèse de doctorat, Université de Nantes, Nantes.

SECORD, James A. [2004], Knowledge in transit, Isis, 95(4), 654-672, doi : $10.1086 / 430657$.

Simon, Josep [2013], Cross-national and comparative history of science education : An introduction, Science 83 Education, 22(4), 763-768, doi : 10.1007/s11191-013-9576-7.

Simon, Josep \& Herran, Néstor [2008], Introduction, dans Beyond Borders : Fresh perspectives in history of science, édité par J. Simon, N. Herran, T. Lanuza-Navarro, P. Ruiz-Castell \& X. Guillem-Llobat, Cambridge : Cambridge Scholars Press, 291-299. 